\title{
A Mathematical Model Describing Consequences of Abnormally High Levels of Epidermal Growth Factor Receptor on the Proliferation of Neoplastic Cells
}

\author{
Michael Pollak, M.D.,* Abraham Boyarsky, Ph.D., $†$ \\ and Pawel Gora, Ph.D.‡
}

"Departments of Medicine and Oncology

McGill University

and Lady Davis Research Institute of the Jewish General Hospital

3755 Cote St. Catherine Road

Montreal, Quebec, Canada H3T 1 E2

†Department of Mathematics

Concordia University

Montreal, Canada H4B 1R6

$\ddagger$ Department of Mathematics

Warsaw University

Warsaw, Poland

\begin{abstract}
Recent laboratory and clinical data suggest that some human neoplasms exhibit unusually high levels of cell-surface receptors for epidermal growth factor, and that this abnormality is associated with rapid cellular proliferation and poor prognosis. We propose that the existence of an abnormally high number of mitogen receptors is not merely correlated with rapid proliferation but is pathophysiologically responsible for such behavior. Cells with high levels of mitogen receptors may be rendered 'hypersensitive' to mitogenic stimuli, and hence may be stimulated to divide even when ambient mitogen concentrations are at a low 'background' level, insufficient to prompt the division of cells with a normal number of receptors. To investigate this hypothesis further, we have developed a mathematical model that describes proliferative behavior of cells as a function of mitogen concentration and receptor number. The model enables us to simulate the proliferative behavior of cells with various receptor levels at various mitogen concentrations and predicts a growth
\end{abstract}


advantage associated with excess mitogen receptors. Computer simulations based on the model are consistent with previously published experimental data. This work provides support for the view that overexpression of genes encoding normal growth factor receptors can contribute to the inappropriate proliferation of neoplastic cells.

\section{INTRODUCTION}

It is generally recognized that peptide growth factors are involved in the control of normal cellular proliferation, and that derangements of the physiological systems involved in mitogenic signal transduction can result in or contribute to neoplastic behavior $(1,2)$. A well-known example of such a derangement is the uncontrolled growth that results if a cell produces in an uncontrolled autocrine fashion a growth factor for which it bears receptors (3). We present a theoretical analysis of the consequences of another common and potentially important derangement of the signal transduction pathways involved in regulation of proliferation, namely the presence of abnormally high levels of mitogen receptors on neoplastic cells.

Recent experimental work has shown that certain cancer cells cultured in vitro exhibit abnormally high levels of cell-surface epidermal growth factor (EGF) receptors, relative to normal cells (4-7). The basis of this abnormality has been studied in the case of certain squamous and breast tumor cell lines, and has been found to be due to amplification and overexpression of the gene encoding the EGF receptor $(4,5,8)$. The proliferation of clones of cells that either exhibit or lack this abnormality has been measured and at physiological concentrations of EGF, and those cells with elevated receptor number proliferate more rapidly (9-11).

Recent studies suggest that these laboratory observations may have relevance to clinical oncology. Several reports (12-14) indicate that various human tumors exhibit abnormally high EGF receptor levels. It has been observed that the prognosis of patients with tumors bearing abnormally high levels of EGF receptors tends to be poorer than those with lower levels of the receptor (14). Other studies show that elevated EGF receptor level correlates negatively with previously described markers of good prognosis $(15,16)$ and correlates positively with markers of poor prognosis (13).

In order to study the hypothesis that mutations that lead to excess numbers of normal mitogen receptors are pathophysiologically responsible for accelerated and unregulated cellular proliferation, we have developed a mathematical model relating proliferation of cells under idealized conditions to ambient EGF concentration and to EGF receptor level, and used it to simulate the proliferation of neoplastic cells.

\section{METHODS}

Equations describing the model are given in the Appendix. We restrict the model to an idealized situation where the probability of cell division is a function of the number of occupied mitogen receptors, and postulate that when a certain threshold of occupied receptors is reached, cell division will follow. The number of occupied receptors is a function of the mitogen concentration and the receptor number per cell. The growth advantage resulting from the presence of excess receptors was computed as shown in the Appendix (Fig. 1).

Subsequent simulations of changes in tumor size over time under various conditons also are idealized, and exclude factors such as cell death. However, as shown in the Appendix, we do not assume that all cells of the tumor proliferate equally, as it is well recognized that only a small proportion of the cells comprising the tumor have 'stem cell'-proliferative potential, and that nonspecific factors such as nutrient supply restrict the number of actively proliferating cells even further. Although we limit the proportion of cells of the tumor that are proliferating, in this simulation we made no attempt to model in detail other aspects of stem cell behavior, such as the limited proliferative potential of 'transitional' cells.

\section{RESULTS}

Figure 1 shows the relationship the model predicts between the growth advantage of one cell type over another and mitogen concentration. The two cell types differ only in that one cell type has a 100-fold increase in the number of mitogen receptors present. The 'growth advantage' is defined as the ratio of the proliferative rates of the two types of cells. The simulation shows that under conditions of low mitogen concentration, the cell type with excess mitogen receptors has a growth advantage over the normal cells, and that this growth advantage diminishes with increasing mitogen concentration. We can interpret 


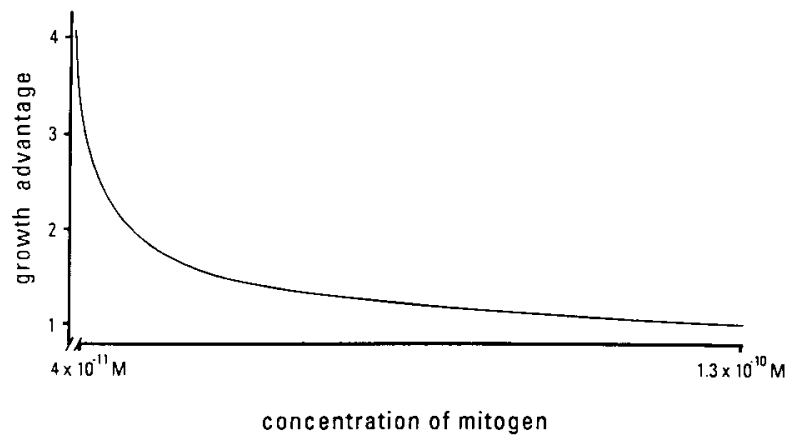

Figure 1. Results of a simulation that compare the rate of proliferation of cells with $5 \times 10^{6}$ EGF receptors to cells with $2 \times 10^{4}$ EGF receptors at various EGF concentrations. This result is interpreted as showing that excess receptor number confers "hypersensitivity" to low concentrations of mitogen that are normally insufficient to trigger cellular division. As the concentration of mitogen increases, the proliferation of the low receptor cell type increases and the growth advantage associated with excess receptors decreases.

this result as showing that the excess receptor number confers 'hypersensitivity' to low concentrations of mitogen that are normally insufficient to trigger cellular division. As the concentration of mitogen increases, the proliferation of the low receptor cell type increases, and the growth advantage associated with excess receptors decreases.

Figure 2 shows simulated growth of tumors comprised of cells identical except for differences in receptor number, at low ambient mitogen concentrations. This simulation demonstrates a growth advantage associated with excess receptors (curve $A, 10^{6}$ receptors vs. curve $\mathrm{B}, 10^{4}$ receptors, at and EGF concentration of $4.5 \times$ $\left.10^{-11} \mathrm{M}\right)$.

Figure 3 illustrates the results of simulation of the growth of identical tumors at varying mitogen concentrations. Note that the model realistically predicts a plateau in dose-response. The simulation illustrates growth at 0.4 $\times 10^{-10} \mathrm{M}$ EGF (curve C), $0.6 \times 10^{-10} \mathrm{M}$ (curve B), and $0.8 \times 10^{-10} \mathrm{M}$ (curve $\mathrm{A}$ ), for cells with $10^{4}$ receptors.

Figure 4 shows how simulated proliferation of a tumor with $10^{4}$ receptors/cell (curve $B$ ). Curve $A$ shows the overall proliferation of the tumor would increase if a single cell with double the receptor number appeared at 7 days. The clone with extra receptors expands more rapidly, so that an increasing proportion of the tumor is comprised of cells with elevated receptor number. The effect is more marked if the receptor number on the single mutated cell increases tenfold, which, biologically, is realistic.

\section{DISCUSSION}

Mutations which lead to the presence of abnormally high levels of functional cell surface EGF receptors are not infrequent in certain kinds of malignancy. To study the pathophysiological consequences of such a derangement, we have developed an idealized model that assumes that when a 'threshold' number of occupied receptors is achieved, cell division will follow. The criterion we use to compare proliferative responses of different cells is the time until the threshold occupancy level is reached. We have excluded from the model a variety of factors that determine cell proliferation, including the action of multiple mitogens and growth inhibitors, nutritional factors, cell density, the varying proliferative potential of individual cells comprising a tumor, and so on. No attempt is made to model the action of mitogens at various specific steps of the cell cycle. Finally, the model is deterministic rather than stochastic, although it would be more realistic

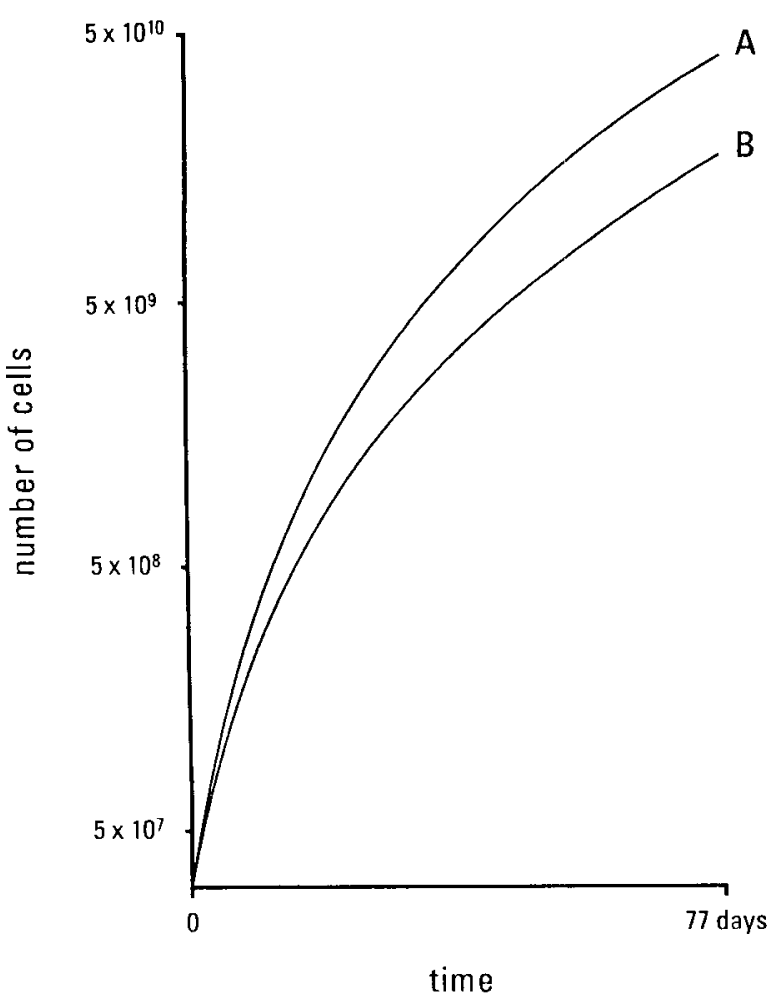

Figure 2. Simulated growth of two tumors comprised of cells identical except for differences in receptor numbers, at low ambient mitogen concentrations. The simulation demonstrates a growth advantage associated with excess receptors (curve A, $10^{6}$ receptors; curve B, $10^{4}$ receptors; mitogen concentration $4.5 \times 10^{-11} \mathrm{M}$ ). 


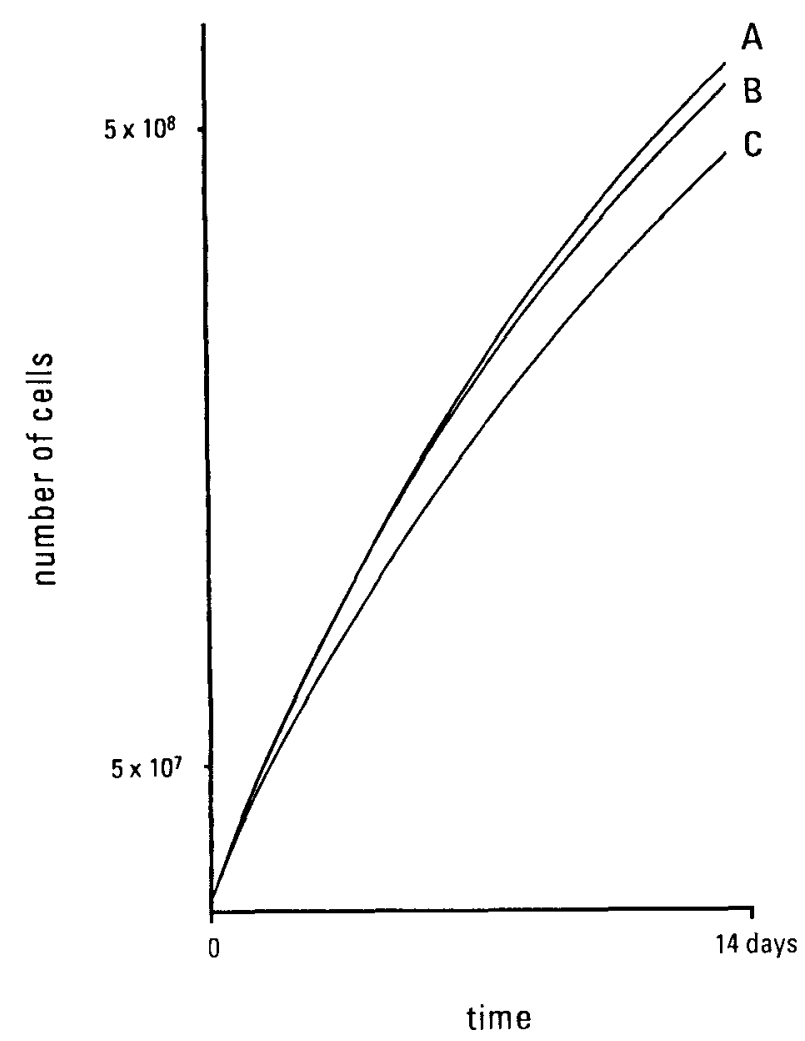

Figure 3. Simulation of the growth of identical tumors at varying mitogen concentrations. Note that the model realistically predicts a plateau in dose/response. The simulation illustrates growth at $0.4 \times$ $10^{-10} \mathrm{M}$ (curve C), $0.6 \times 10^{-10} \mathrm{M}$ (curve B), and $0.8 \times 10^{-10} \mathrm{M}$ (curve A), for cells with $10^{4}$ receptors.

to conceive of increasing probability of cell division with mitogenic stimulation. However, the simplifying assumptions are appropriate to allow us to analyze specifically the effect of the derangement under consideration, namely the presence of excess mitogen receptors.

The simulations give results consistent with the concept that the presence of excess mitogen receptor can confer 'hypersensitivity' to mitogenic stimulation. Under conditions of low ambient mitogen concentration, it is possible that such hypersensitivity will lead to stimulation of proliferation of cells with excess receptors, while normal cells remain unstimulated.

Available data would suggest that the appearance of excess numbers of mitogen receptors is more likely to be a "progression" event rather than a "carcinogenic" event. While on theoretical grounds, the appearance of excess receptors might be sufficient in itself to cause neoplastic behavior, there are no documented examples of this. However, in the case of gliomas (17) it appears that the increasingly abnormal proliferation of tumor cells over time may be the result of the appearance in the tumor of malignant clones bearing excess numbers of EGF receptors. From the point of view of the individual tumor cell, the presence of excess receptors represents an adaptive mutation that allows rapid proliferation under conditions where this would not normally take place. However, from the point of view of the host, the mutation represents an escape from the normal mechanisms that serve to regulate cellular proliferation.

The existence of a class of neoplasms that exhibits 'hypersensitivity' to mitogenic stimulation may have therapeutic implications for new hormonal forms of treatment. Tumors with such derangements would preferentially bind competitive antagonists to mitogen receptors, or mitogen-linked toxins (18). Such treatment can, on

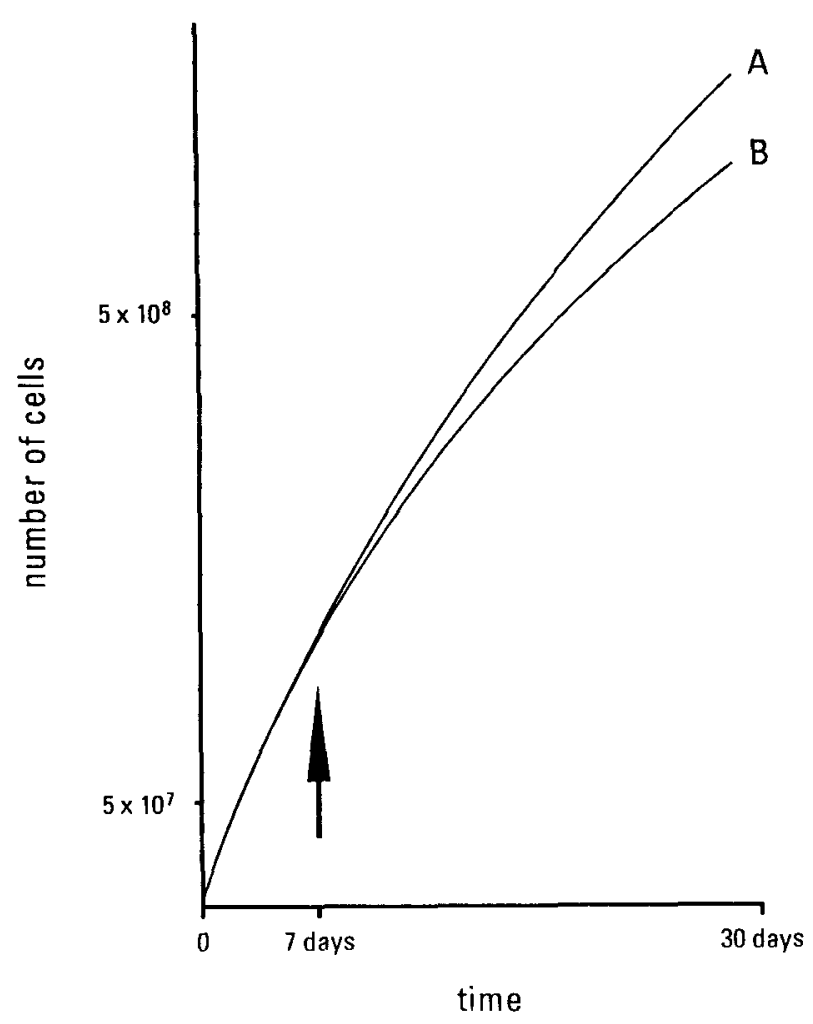

Figure 4. Simulated proliferation of a tumor with $10^{4}$ receptors/cell (curve B). Curve A shows how the overall proliferation of the tumors would increase if a single cell within double the receptor number appears at 7 days. The clone with extra receptors expands more rapidly, so that an increasing proportion of the tumor is comprised of cells with elevated receptor number. The effect is more marked if the receptor number on the single mutated cell increases 10 -fold, which is biologically realistic. 
theoretical grounds, be predicted to preferentially effect cells with excess receptors, as compared to normal tissues. Recent work with animal models is consistent with this prediction (19). In a similar fashion, efforts at synchronizing and stimulating neoplastic cell proliferation with peptide mitogens to enhance the efficacy of cytotoxic treatment might be particularly effective for tumors that exhibit hypersensitivity to mitogenic stimulation relative to normal tissue. This theoretical analysis encourages further experimental work in this area.

\section{ACKNOWLEDGMENTS}

Supported by grants to MP from the Cancer Research Society of Montreal and to $\mathrm{AB}$ from the National Sciences and Engineering Research Council of Canada and Fondes pour la Formation de Chercheurs et L'aide a la Recherche du Quebec.

\section{APPENDIX}

\section{Mitogenic Simulation}

Definitions: Let $u$ be the basic unit of length. Let $p$ be the number of cell receptors $/ u^{2}$ at time $t=0$. Let $x(t)$ denote the number of receptors $/ u^{2}$ that are occupied at time $t$ after exposure to the mitogen. Let $c$ be the concentration of mitogen in the cell environment, that is, $c$ is the number of mitogen molecules $/ u^{3}$.

We shall now write an equation describing the dynamics of $x(t)$ :

$$
\frac{d \mathrm{x}(t)}{d t}=A c\left(1-\frac{x(t)}{p}\right)
$$

where $A$ is a proportionality constant, For $x(t)$ very small, the rate of change depends only on $c$. This says that initially the rate at which receptor sites are being occupied by mitogen molecules depends only on the concentration of mitogen. This assumption may be reasonable for certain ranges of $p$. Once $x(t)$ becomes significant, $p$ enters the formula and the rate of increase of occupied receptor sites decreases much more rapidly for small $p$ than for large $p$. We remark that $A$ and $p$ can only be taken as constants (for a given cell) if the total cell surface receptor number does not change (e.g., be EGF-induced internalization and down regulation) during the experiment. This is questionable if we observe the response (20).

A more realistic dynamical model is described by:

$$
\frac{d x(t)}{d t}=A c\left(1-\frac{x(t)}{p}\right)-B x(t)
$$

The second term on the right-hand side of (2) reflects dissociation. We remark that (2) is not the standard chemical kinetic equation in that the parameter $A$ is constant, and not proportional to the receptor density $p$, where $A=k_{t} p$. Equation (2) assumes that the forward reaction rate is effectively independent of $p$, the receptor density, for low concentrations of mitogen. We suggest that this is an intuitively reasonable assumption since when the mitogen concentration is small, the relatively few mitogen molecules are attracted to the cell receptors at a rate which does not depend on the total number of receptors. Furthermore, in the sequel we shall show that for low mitogen concentrations, Eq. (2) models observed data while the standard model cannot.

We rewrite (2) as:

$$
\frac{d x(t)}{d t}+\left(\frac{A c}{p}+B\right) x(t)=A c
$$

This is a first-order linear differential equation whose solution is

$$
x(t)=\frac{A c}{B+A c / p}[1-\exp (-(B+A c / p) t)]
$$

Definitions: Let $X$ denote the number of total receptors necessary to trigger replication of the cell. Let $T$ be the time at which $X$ receptors are occupied. Let $X_{c}$ be the number of occupied receptors $/ u^{2}$ necessary to trigger replication of the cell. Solving for $T$ in Eq. (3), we obtain:

$$
T=\frac{-p}{A c+B p} \ln \left[1-\frac{A c+B p}{A c p} X_{c}\right]
$$

Now let $p_{1}$ and $p_{2}\left(p_{1}<p_{2}\right)$ be two numbers of unoccupied receptor sites for two different cell types. We are interested in determining the growth advantage of these cell types with respect to each other as a function of $c$. To do this, we compute:

$$
\frac{T_{1}}{T_{2}}=\frac{\frac{-p_{1}}{A c+B p_{1}} \ln \left[1-\frac{A c+B p_{\mathbb{1}}}{A c p_{1}} X_{c}\right]}{\frac{-p_{2}}{A c+B p_{2}} \ln \left[1-\frac{A c+B p_{2}}{A c p_{2}} X_{c}\right]}
$$


Using the formula for the growth advantage $T_{1} / T_{2}$ deduced from the model, we can prove two facts which are in agreement with experimental data:

(i) $T_{1} T_{2} \cong 1$ for very high concentration $c$ of mitogen molecules;

(ii) $T_{1} / T_{2}$ is decreasing as a function of $c$ (at least for large values of $c$ ), which means that the smaller the concentration $c$ is, the bigger the growth advantage $T_{1} / T_{2}$ will become. It should be mentioned here that the computer plottings of $T_{1} / T_{2}$ show that it is a decreasing function of $c$, for all admissible $c$.

Let us introduce the notation:

$$
S_{1}=\frac{A c+B p_{1}}{A c p_{1}} \quad \text { and } \quad S_{2}=\frac{A c+B p_{2}}{A c p_{2}}
$$

In this notation,

$$
\frac{T_{1}}{T_{2}}=\frac{S_{2} \ln \left(1-S_{1} X_{c}\right)}{S_{1} \ln \left(1-S_{2} X_{c}\right)}
$$

When $c$ is very large, then $S_{1}$ and $S_{2}$ become very small and we can use the approximate formula $\ln (1-w)$ $\cong-w$, obtaining $T_{1} / T_{2} \cong 1$, for large $c$, which proves (i).

To prove (ii), we consider the derivative $d\left(T_{1} / T_{2}\right) / d c$. After quite complicated, but elementary calculation we get

$$
\frac{d\left(T_{1} / T_{2}\right)}{d c}=\frac{B S_{1} \ln Z_{2}}{c^{2} A S_{2} \ln Z_{1}}\left[\frac{1}{S_{2}}-\frac{1}{S_{1}}+X_{c}\left(\frac{1}{Z_{2} \ln Z_{2}}-\frac{1}{Z_{1} \ln Z_{1}}\right)\right]
$$

where $Z_{1}=1-S_{1} X_{c}, Z_{2}=1-S_{2} X_{2}$

Assuming $P_{1}, P_{2}$, and $c$ are big enough, we can once again use the approximation $\ln (1-w) \cong-w$ to obtain

$$
\frac{d\left(T_{1} / T_{2}\right)}{d c}=\frac{B S_{1} \ln Z_{2}}{c^{2} A S_{2} \ln Z_{1}}\left[\frac{1}{Z_{2}}-\frac{1}{Z_{1}}\right]
$$

which is negative for $p_{2}>p_{1}$. Thus if $p_{2}>p_{1}$, the growth advantage $T_{1} / T_{2}$ is decreasing as a function of $c$, for $c$ large.

Formula (5) can be rewritten in the form

$$
\frac{T_{1}}{T_{2}}=\frac{\left(1 / p_{2}+D\right) \ln \left(1-X_{d} / p_{1}-D X_{c}\right)}{\left(1 / p_{1}+D\right) \ln \left(1-X_{d} / p_{2}-D X_{c}\right)}
$$

where $D=B / A c$. Note that if $c$ increases, then $D$ decreases.
Using formula (7) we can plot $T_{1} / T_{2}$ as a function of $D$ and, therefore, as a function of $c$. Let us consider a cell type having a radius of 10 microns. Then the surface area of the cell is given by $4 \pi\left(10^{-5}\right)^{2} \mathrm{~m}^{2}$ or $4 \pi 10^{-6} \mathrm{~cm}^{2}$. If there are $P$ receptors on the cell, then the concentration of receptors $/ \mathrm{cm}^{2}$ is $p=P 10^{6 / 4 \pi}$. The concentration of occupied receptors necessary to trigger replication is $X_{c}=X \times 10^{6 / 4 \pi}$ per $\mathrm{cm}^{2}$. For $P_{1}=10^{4}, P_{2}=$ $10^{6}, X=5 \times 10^{3}$. Figure 1 shows $T_{1} / T_{2}$ as a function of concentration of mitogen $c$. As $c$ increases $T_{1} / T_{2}$ approaches a number close to 1 . In words this says that for large concentration of mitogen, the time it takes to occupy $X$ receptors is independent of $P$. However, when $c$ is small, the times to occupy $X$ receptors depends critically on $P$.

\section{Discussion of Standard Kinetic Model}

If we had used the standard kinetic model

$$
\frac{d x(t)}{d t}=k_{t} p c\left(1-\frac{x(t)}{p}\right)-B x(t)
$$

where $k_{t} p$ replaces $A$ in (2), $k+$ being the forward rate constant for the binding of EGF in solution to EGF receptors on the cell surface, the expression for the time $T$ at which $X$ receptors are occupied is given by

$$
T=\frac{-1}{k_{t} p+B} \ln \left[1-\frac{k_{t} c+B}{k_{t} p c} X_{c}\right]
$$

Although this expression for $T$ is well-defined for $c$ large, there is a problem for $c \rightarrow 0$, since the argument in the $\log$ term becomes negative at $a$ finite $c$. This difficulty is again encountered in the formula for growth advantage:

$$
\frac{T_{1}}{T_{2}}=\frac{\ln \left[1-\frac{k_{t} c+B}{k_{t} c p_{1}} X_{c}\right]}{\ln \left[1-\frac{k_{t} c+B}{k_{t} c p_{2}} X_{c}\right]}
$$

For the foregoing data: $p_{1}=10^{10 / 4 \pi}, p_{2}=10^{12 / 4 \pi,} X_{c}$ $=5 \times 10^{9 / 4 \pi}$ and $T_{1} / T_{2}=4$, we have:

$$
\frac{\ln (1-E / 2)}{\ln (1-E / 200)}=4
$$


where $E=1+B / k_{t} c$. Equation (11) can only be true if $E<0$. But this implies that $B / k_{t} c<0$, which is impossible. Furthermore, from (10), we have

$$
\lim _{c \rightarrow \infty} T_{1} / T_{2}=\frac{\ln \left(1-X_{c} / p_{1}\right)}{\ln \left(1-X_{c} / p_{2}\right)}
$$

For the values of $X_{c}, p_{1}$, and $p_{2}$ used above, this limit is 139 . But experimentally we know that $T_{1} / T_{2}$ tends to 1 ac $c \rightarrow \infty$. model. Hence the standard model for chemical kinetics is not appropriate for the experimental data we are considering.

\section{Tumor Growth}

Let $r$ denote the radius of a cell and let $R$ be the radius of a tumor, considered as a sphere. Then (4/3) $\pi R^{3}$ is the volume of the tumor and (4/3) $\pi r^{3}$ is the volume of a cell inside the tumor. Hence there are $(R / r)^{3}$ cells in the tumor. We assume that only the $m$ outer layers of the tumor are exposed to sufficient mitogen concentrations which allows them to contribute to tumor growth. The volume of the $m$ outer layers of the tumor is

$$
\begin{aligned}
(4 / 3) \pi R^{3}-(4 / 3) \pi(R-m r)^{3} & = \\
(4 / 3) \pi\left(3 R_{2} m r-3 R m^{2} r^{2}\right. & \left.+m^{3} r^{3}\right)
\end{aligned}
$$

Hence there are $e=\left(3 R^{2} m r-3 R m^{2} r^{2}+m^{3} r^{3}\right) / r^{3}$ cells in the $m$ outer layers of the tumor. Since there are $(R / r)^{3}$ cells in the tumor.

$$
b=e /(R / r)^{3}=\left(3 R^{2} m r-3 R m^{2} r^{2}+m^{3} r^{3}\right) / R^{3}
$$

is the fraction of all cells in the tumor which are dividing. Let $y(n)$ denote the number of cells in the tumor at time $n$. Since each cells becomes two cells after division, the $(n+1)$ th generation will consist of $2 \mathrm{~b}$ times the number of cells in the $n$-th generation plus $(1-b)$ ) times the number of cells in the $n$-th generation. Mathematically, this is written as:

$$
\begin{aligned}
y(n+1) & =2 b y(n)+(1-b) y(n) \\
& =(1+b) y(n)
\end{aligned}
$$

where the integer $\mathrm{n}$ denotes the $\boldsymbol{n}$-th generation after observation commences. On substituting for $b$, we get

$$
y(n+1)=y(n)+\frac{3 R^{2} m r-3 R m^{2} r^{2}+m^{3} r^{3}}{R^{3}} y(n)
$$

Since the volume of the tumor at time $n$ is equal to $y(n)$ the volume of each cell, we have

$$
(4 / 3) \pi R^{3}=y(n)(4 / 3) \pi r^{3}
$$

and therefore the $R$, the radius of the tumor at time $n$ generations is $r y(n)^{1 / 3}$. Substituting this into (12), we obtain

$$
y(n+1)=y(n)+3 m y(n)^{2 / 3}-3 m^{2} y(n)^{1 / 3}+m^{3}
$$

For most cases of interest, $m$ is small compared to $y(n)$ and $y(n)$ is large. Under these conditions, (13) simplifies to

$$
y(n+1)=y(n)+3 m y(n)^{2 / 3}
$$

The time between the divisions is the sum of two times: the time $T$ needed to fill up $X$ receptors and the time, $\phi$, to divide after $X$ receptors are occupied. We assume that $\phi$ is constant and equals approximately 8 hours. Hence the time between consecutive divisions is $T_{g}=T+\phi$ and it depends on the number of receptors $P$, the concentration of mitogen $c$ and the number $X$ of receptors which have to be occupied to trigger the division.

The growth of the number of cells in the tumor is described by the equation

$$
y\left((n+1) T_{g}\right)=y\left(n T_{g}\right)+3 m y\left(n T_{g}\right)^{2 / 3} .
$$

To find an explicit formula for the number of cells at time $t, y(t)$, we consider the differential equation

$$
\frac{d \bar{y}}{d n}=3 m(\bar{y})^{2 / 3},
$$

which corresponds to the difference Eq. (14). Its solution is

$$
\mathrm{y}(n)=(m n+\sqrt[3]{y(0)})^{3}, n \in \mathbb{R}
$$

which is a good approximation to $y(n)$ obtained from formula (14).

Using (15), we obtain the following approximate formula for the number of cells at time $t$ :

$$
\mathrm{y}(t)=\left(m \frac{t}{T_{g}}+\sqrt[3]{y(0)}\right)^{3}
$$


For $y(0)=3 \times 10^{7}$, Figure 2 shows the growth curves for different times $T_{1}$ and $T_{2}$, i.e., for cells with different numbers of receptors $P_{1}$ and $P_{2}$, respectively.

Using (4), we can now express $y(t)$ as a function of mitogen concentration and the number of receptor sites:

$y(t)=\left\{\frac{m t}{\phi-\frac{p}{A c+B p} \ln \left(1-\frac{A c+B p}{A c p} X_{c}\right)}+\sqrt[3]{y(0)}\right\}^{3}$

To estimate the constants $A$ and $B$, we proceed as follows. From experimental data (10) we know that reasonable estimates are $T_{1} / T_{2} \cong 4$ at $c=4.5 \times$ $10^{-11} \mathrm{~m}$, and $P_{1}=10^{4}, P_{2}=10^{6}$. Using Eq. (7), we allowed $D$ to vary until the right-hand side of Eq. (7) equaled 4. This occurred for $B / A=2.18$. Next, we approximated the growth curves of Figure 5 of (10) by using our model with $T_{1}=20 \mathrm{~min}, T_{2}=80 \mathrm{~min}$. Using $B / A=2.18, T_{1}=20 \mathrm{~min}, c=4.5 \times 10^{-11} \mathrm{M}$ and $P_{1}$ $=10^{4}$ in Eq. (4) yields $A=1.8$ and $B=3.95$.

\section{REFERENCES}

1. Goustin AS, Leof EB, Shipley GD, Moses HL: Growth factors and cancer. Cancer Res 46:1015-1029, 1986.

2. Buick RN, Pollak MN: Perspectives on clonogenic tumor cells, stem cells, and oncogenes. Cancer Res 44:4904-4918, 1984.

3. Sporn MB, Roberts AB: Autocrine growth factors and cancer. Nature 313:745-747, 1985.

4. Filmus J, Pollak MN, Cailleau R, Builk RN: MDA-468, a human breast cancer cell line with a high number of epidermal growth factor (EGF) receptor, has an amplified EGF receptor gene and is growth inhibited by EGF. Biochem Biophys Res Comm 128:898-905, 1985.

5. Filmus J, Pollak MN, Cairncross JG, Buick RN: Amplified, overexpressed and rearranged epidermal growth factor receptor gene in a human astrocytoma cell line. Biochem Biophys Res Comm 131:207-215, 1985.

6. Fabrikant RN, Delarco JE, Todaro GJ: Nerve growth factor receptors on human melanoma cells in culture. Proc Natl Acad Sci (USA) 74:565-569, 1977.
7. Haigler H, Ash JF, Singer SJ, Cohen S: Visualization by fluorescence of the binding and internalization of human carcinoma cells A431. Proc Natl Acad Sci (USA) 75:3317-3321, 1978.

8. Lin CR, Chen WS, Kruiger W et al: Expression cloning of human EGF receptor cDNA: gene amplification and 3 related mRNA products in A431 cells. Science 224:843-848, 1984.

9. Kawamoto T, Mendelsohn J, Le A et al: Relation of EGF receptor concentration to growth of human epidermal A431 cells. J Biol Chem 259:7761-7766, 1984.

10. Filmus J, Trent JM, Pollak MN, Buick RN: Epidermal growth factor receptor gene-amplified MDA-468 breast cancer cell line and its nonamplified variants. Mol Cell Biol 7:251-257, 1987.

11. Santon JB, Cronin MT, Maclood CL et al: Effects of EGF receptor concentration on tumors of A431 cells in nude mice. Cancer Res 46:4701-4705, 1986.

12. Ro J, North SM, Gallick GE et al: Amplified and overexpressed EGF receptor gene in uncultured primary human breast carcinoma. Cancer Res 48:161-164, 1988.

13. Spitzer E, Grosse R, Kunde D, Schmidt HE: Growth of mammary epithelial cells in breast cancer biopsies correlates with EGF binding. Int J Cancer 39:279-282, 1987.

14. Sainsbury JRC, Rarndon JR, Needham GR et al: Epidermalgrowth-factor receptor status as predictor of early recurrence of and death from breast cancer. Lancet 1:1398-1402, 1987.

15. Perez R, Pascual M, Macias A, Lage A: Epidermal growth factor receptors in human breast cancer. Breast Cancer Res Treat 4:189-193, 1984.

16. Fitzpatrick SL, Brightwell J, Wittliff JL et al: Epidermal growth factor binding by breast tumor biopsies and relationship to estrogen and progestin receptor levels. Cancer Res 44:3448-3453, 1984.

17. Libermann TA, Nusbaum HR, Razon et al. Amplification enhanced expression and possible rearrangement of EGF receptor gene in primary human brain tumors of glial origin. Nature 313:144-147, 1985.

18. Vollmar AM, Banker DE Medelsohn J, Herschman HR: Toxicity of ligand and antibody directed ricin A-chain conjugates recognizing the epidermal growth factor receptor. J Cell Physiol 131:418-425, 1987.

19. Goldenberg A, Masui H, Divgi $C$ et al: Imaging of human tumor xerografts with indium 111-labelled anti-epidermal growth factor receptor monoclonal antibodies. J Natl Cancer Inst 81:1616-1625, 1989.

20. Khauer DJ, Wiley HS, Cunninham DD: Relationship between epidermal growth factor receptor occupancy and mitogenic response. J Biol Chem 259:5623-5661, 1984. 